\title{
Peri-tumoral Lymphovascular Density by Antipodoplanin Antibody D2-40, as a Predictor of Nodal Metastasis in Oral Squamous Cell Carcinoma
}

\begin{abstract}
BACKGROUND:

Involvement of regional lymph nodes by cancer is a major determinant of prognosis in oral squamous cell carcinoma (OSCC) and is a significant predictor of patient survival. Peri-tumoral lymphangiogenesis is being studied as an important tool in predicting neck lymph node metastasis. Podoplanin, a transmembrane glycoprotein is expressed on lymphatic vessel endothelium and considered as a marker of lymphangiogenesis. High podoplanin expression has been associated with invasion, progression and metastasis of oral cancer. D2-40, an antipodoplanin monoclonal antibody, is specific marker of lymphatic endothelial cells. The present study was designed to investigate the peritumoral lymphovascular density (PT-LVD) by evaluating D2-40 expression and its potential utility in predicting lymph node metastasis in OSCC.

METHODS: Seventy two patients with OSCC were enrolled (36 with early and 36 with advanced stage tumors). Differential expression of peritumoral lymphovascular density (PT-LVD) was evaluated using D2-40 expression by immunohistochemistry (IHC). Expression was categorized as low and high, derived from mean of number of vessels per 10 high power fields in hot-spots. Statistical methods were employed to correlate expression with age, gender, tumor site, stage and neck lymph node involvement.

RESULTS: We observed a significant association between peritumoral lymphovascular density (PT-LVD) with clinical neck lymph node status $(p=0.001)$ and tumor stage $(p=0.033)$. There was no significant correlation of D2-40 expression with age, gender, tumor site, $T$ stage and tumor differentiation.

CONCLUSION: PT-LVD is a positive predictor of neck lymph node metastasis. It has translational potential, along with other clinical parameters for decision making in treatment of neck in oral cancer.
\end{abstract}

Keywords: Oral carcinoma, podoplanin, D2-40, lymphovascular density, oral squamous cell carcinoma, neck metastasis

\section{INTRODUCTION}

Head and neck squamous cell carcinoma (HNSCC) is a major public health problem in India. In contrast to the Western population, where this malignancy is the 6th most common cancer, in India, HNSCC represents the most frequent cancer in males.[1] Oral squamous cell carcinoma (OSCC), the most common subtype of HNSCC, constitutes $40-70 \%$ of all diagnosed HNSCC cases.[2] Cigarette smoking and smokeless form of tobacco consumption is the predominant cause of these cancers.[3]
Kunwar Parvez MBBS1, MS, Vipin Arora

MS, FICS 2 \& Neelam Wadhwa MD 3

1Senior Resident, University College of Medical Sciences \& GTB Hospital, Dilshad Garden, Delhi, 110095 INDIA

2 Professor of ENT and Head Neck Surgery, University College of Medical Sciences \& GTB Hospital

3 Professor Pathology, University College of Medical Sciences \& GTB Hospital

E mail: vipinar@gmail.com

Cite as: Parvez, K., Arora, V., Wadhwa, N. (2020) Peri-tumoral Lymphovascular density by antipodoplanin antibody D2-40, as a predictor of nodal metastasis in oral squamous cell carcinoma. The Physician vol 6: Issue 3: epub 27.11.2020 DOI: https://doi.org/10.38192/1.6.3.6

Article Information

Submitted 21.10.2020

EpUB 27.11.2020

Open access- Creative commons Licence v4 (CC-BY - ND-4.0)

Involvement of regional lymph nodes by cancer is a major determinant of the prognosis of OSCC and is a significant predictor of survival. The presence of lymph node metastases $(\mathrm{N}+)$ is associated with $50 \%$ reduction of the 5-year survival rate, irrespective of the treatment modality employed.[4,5] Clinically, lymph nodes are assessed by physical examination, CT Scan, utrasonography and magnetic resonance 
imaging, which have variable sensitivity and specificity.[6] While these modalities are successful in detecting an obvious metastasis, occult metastatic spread may often be missed. Accurate assessment of lymph node metastasis is important for deciding the treatment modality. Presence of metastasis warrants neck dissection which is associated with high morbidity. Advanced oral primary malignancies (T3, T4) with clinically uninvolved cervical lymph nodes (cNO) are indications for elective neck dissection, due to high incidence of occult metastasis in these cases. Studies have shown that the incidence of occult neck metastases in patients with stage I/II disease may be as high as $42 \%$.[7]

Typically, $\mathrm{T} 1$ and $\mathrm{T} 2$ lesions are associated with a risk of regional metastasis of $10 \%$ to $30 \%$ respectively. Elective neck dissection in these patients may result in overtreatment of approximately two thirds of patients who do not have neck metastasis but had to undergo an unnecessary surgery associated with increased morbidity. On the other hand, deciding against neck dissection may miss occult metastasis in a large subset of patients who are undertreated, leading to a poor overall survival rates. $[7,8]$

Currently, there is no reliable method to preoperatively predict the lymph node metastasis, so that patients who are at higher risk of neck node metastasis could be selected and treated by neck dissection, while sparing the patient with low risk from undergoing an unnecessary highly invasive surgical procedure.

Head neck cancers are known to augment local lymphangiogenesis, which is associated with increased lymphatic spread and cervical lymph node metastasis.[9] Tumor-induced lymphangiogenesis is mediated by lymphangiogenic growth factors such as vascular endothelial growth factor C (VEGF-C), which are produced and secreted by the tumor themselves, stromal cells, tumor-infiltrating macrophages, or activated platelets.[10-12] These factors induce lymphangiogenesis via enhancing expression of podoplanin within the tumor, in its surroundings and tumor-draining lymph node prior to metastatic tumor dissemination. The expression levels of podoplanin are further elevated upon seeding of the metastatic cells and associated with inreased intra-tumoral lymphnagiogenesis (ITL) within the tumor mass and peri-tumoral lympangiogenesis (PTL) in the tumor periphery. While PTL is the main conduit of metastasis[13], intratumor lymphatics are mostly deformed and are believed to be not involved in the spread of cancer cells.[14,15] Enlarged lymphatic vessels that drain the tumor enhance the delivery of tumor cells to the lymph node most likely via enhanced lymphatic flow rates and facilitation of metastatic tumor cell cluster dissemination.[16-18]

Podoplanin is a 38-kd mucin type transmembrane glycoprotein that is specifically expressed in lymphatic endothelium. Podoplanin expression correlates with lymph node metastasis in squamous cell carcinoma of the oral cavity and D2-40 antibody is a marker for M2A antigen of podoplanin.[19] D240 is a very reliable marker of podoplanin and has high reproducibility for tumor lymphangiogenesis and lymphatic vessel invasion in human cancers. D240 shows specific staining reaction to lymphatic endothelial cells (LEC), but not to the adjacent blood vessel capillaries and hence, can be used to measure lymphovascular density [LVD].[20] D2-40, being a specific marker of LECs, can be used as a predictor of tumor progression by lymphatic metastasis and may be a potentisl therapeutic target for OSCC. Several groups have studied the intratumoral (ITLVD) and peritumoral lymphatic density (PT-LVD) in oral and other head neck cancers. While it was reported that D2-40 is a highly specific marker for LECs.[21,22], there are still conflicting reports on its association with PT-LVD, lymph node metastasis, overall survival, $T$ staging and tumor differentiation. The current study was aimed to evaluate the extend of PT-LVD in oral SCC by assessing D2-40 expression. This study evaluates the predictive value of PT-LVD in real life scenario, in which decision for neck treatment is made pre-operatively based on tumor characteristics and imaging.

\section{MATERIAL AND METHODS}

This is an analytical, cross-sectional study which was conducted in the Department of Otorhinolaryngology, Department of Pathology at University College of Medical Sciences \& GTB Hospital and Delhi State Cancer Institute, Delhi between November 2015 and April 2017. The study was approved by institutional ethics committee. Seventy two patients with squamous cell carcinoma of oral cavity with or without regional cervical lymphadenopathy were included in the study. Thirty six patients recruited had T1 and T2 tumors (early disease), while the remaining 36 patients were diagnosed with T3 and T4 malignancies (locally advanced disease). 
Exclusion criteria: Patients who had received any form of previous treatment such as surgery, radiation and chemotherapy, pregnant patients, poor general condition and were unfit for surgery or refused consent were excluded from the study.

Detailed clinical history, head \& neck and systemic examinations were performed. Contrast enhanced CT scan of the primary and neck for tumor mapping and evaluation of metastatic lymph nodes was done and TNM staging was assigned as per AJCC 7th edition. Patients were categorized as $\mathrm{N}+$ if there was evidence of neck nodal metastasis on physical examination or CT scan. Incisional biopsy from the primary lesion including advancing radial margin with least $1 \mathrm{~mm}$ of the apparently normal tissue was taken.

Histopathology reports were noted. Immunohistochemistry (IHC) was performed for D240 by following technique:

Four micron thick sections were obtained on poly-LLysine coated slides. Sections were de waxed in xylene and rehydrated through graded concentration of alcohol. Antigen retrieval was performed by heating in citrate buffer $(\mathrm{pH} \mathrm{6.0)}$ in a microwave oven for 30 minutes. Endogenous peroxidase activity was blocked with $3 \% \mathrm{H}_{2} \mathrm{O}_{2}$ solution in methanol for 30 minutes, after washing with TRIS buffer solution for $5 \mathrm{~min}$. The primary antibody was applied in a humid chamber at room temperature for 60 minutes and sections washed with TRIS buffer. Secondary \& tertiary antibodies was applied successively for 30 minutes each and washed with TRIS buffer in between. DAB (Diaminobenzidinetetrahydrochloride) was used as a chromogen. All sections were counterstained with haematoxylin.

All IHC slides were interpreted by a single pathologist (NW) and lympho-vascular density in the peritumoral region was calculated (PT-LVD).

Quantitative analysis of the peritumoral lymphatic vessel density was performed in sections that stained for D2-40. The entire tissue section was screened at low (10X) and mid (20X) magnification for identifying hot spots. The lymphatics were identified as thinned wall vessels lined by flattened endothelial cells. The antibody staining was specific as blood capillaries which look similar to lymphatics were DAB negative. Lymphatic density was defined as the mean of number of lymphatic vessels counted in 10 high power optical fields at $200 \mathrm{X}$. The range of LVD (lymphovascular density) noted was 0 to 11 . Cases with LVD less than 2 were considered low LVD and if more than or equal to two then it was considered high LVD.

Statistical Analysis: Chi-square test was used to assess the correlation between D2-40 expression with tumor stage and nodal metastasis. Unpaired student t-test was used to assess the difference between PT-LVD. Data was analyzed by SPSS software version 20. $p$ value of less than 0.05 was considered significant.

\section{Results:}

\section{Association between clinical lymph node and PT-} LVD

A total of 72 patients with oral squamous cell carcinoma were included in the present study. Tumor subsite breakup is summarized in Table 1. Buccal mucosa was the most frequent histological subsite of cancer in our patients' cohort, followed by tongue. While no statistically significant association was observed between tumor differentiation and PT-LVD ( $p=0.177)$ (Table 2), 33 of the 72 cases analyzed displayed clinically positive lymph node, resulting in statistically significant association between PT-LVD and clinical lymph node metastasis in oral carcinoma (Table 3).

\section{Clinical stage and PT-LVD}

Table 4 shows that clinical stage (TNM) of oral SCCS was significantly associated with (PT-LVD) $(p=0.033)$.

\section{Association between primary tumor and peritumoral lymphatic vascular density \\ There were 36 patients in the each group of early primary (T1\&T2) and locally advanced primary tumor (T3\&T4). There was no significant association between group I (T1\&T2) \& group II (T3\&T4) with peritumoral lymphovascular density (Table 5), as well as with the histological subsite of primary tumor (Table 6). Furthermore, there was no significant difference in PT-LVD associated with either with age and gender.}

\section{DISCUSSION}

Lymph node metastasis is one of the most important prognostic factors for HNSCC.[23,24] It is still unclear whether lymphatic dissemination of tumor cells is through newly formed lymphatic vessels by lymphangiogenesis or through pre- 
existing lymphatics. While it has been found that patients with HNSCC have a high number of lymphatic vessels, observed both within the tumor and in peritumoral region.[25], it is still a controversy whether the major contributors for nodal metastasis are intratumoral or peritumoral lymphatic vessels. Franchi et al. have suggested that both are likely to have a role in dissemination.[25] In their study, the authors have reported a correlation between PT-LVD and lymph node involvement. In contrast, a study conducted by Kyzas et al shows a correlation between lymph node involvement and IT- LVD. It was observed that intratumoral lymphatics are comprised of disorganized lymphatic vessels which seriously limit their usefulness for an effective conduit for metastasis, compared with the fully functional peritumoral lymphatic vessels. [26] Therefore we planned to study the peritumoral lymphatic vessel density, using D2-40 in patients with OSCC. In this study we report a significant association between peritumoral LVD with lymph node involvement and clinical stage in patients with oral cancer.

Of the 72 OSCC patients analyzed, most were in $4^{\text {th }}$ decade at the time of diagnosis. The youngest patient was 22 years old while oldest was 72 years of age. Male to female ratio was 9:1. The most common site for oral SCC was buccal mucosa (58.3\%). The most common symptom was nonhealing ulcer $(80 \%)$.These finding are consistent with other studies. [27,28]

33 of 72 patients (45\%) were clinically node positive, which is consistent other Indian studies (Kyzas et al and Watanabe et al report $26 \%$ and $40 \%$ lymph node positive cased respectively) where patients usually present at an more advanced stage due to lack of awareness and healthcare facilities.

Ping Yuan et al reported that high podoplanin expression was associated with higher pathologic stage $(P<0.007)$ and patients whose tumors expressed high levels of podoplanin had a statistically significant higher rate of lymph node metastasis ( $P<.0001)$, but no association with clinical T stage was found. [29] In our cohort of OSCC tumors from different histological subsites (lip, buccal mucosa, tongue, and hard palate), 16 cases (22.2\%) did not stain with D2-40, 30 cases (41.6\%) had low lymphovascular density and 42 cases (58.3\%) expressed high lymphovascular density. While there was no significant association of PT-LVD and primary tumor size (T), a significant association was observed between PT-LVD with lymph node metastasis $(p=0.001)$ and overall clinical stage $(p=0.033)$. In concordance with Yuan et al [29], our study reveals a significant association between clinical lymph node involvement and PT-LVD.

Kyzas et al evaluated whether IT and PT-LVD correlate with presence of lymph node metastasis at the time of diagnosis and with other clinicopathological parameters, including overall survival. They found that high IT-LVD \& PT-LVD were both significantly associated with the presence of lymph node metastasis at the time of diagnosis. [26] Their results are similar to our study as there was a significant association between clinically positive lymph node and PT-LVD. We did not study IT-LVD as this was not the aim of our study.

Franchi et al, [9] quantified lymphangiogenesis within the tumor and peritumoral area in $52 \mathrm{HNSCC}$ specimens, D2-40-positive lymphatic vessels were detected in all cases. They observed that high peritumoral lymphangiogenesis was associated with an increased risk of developing lymph node metastasis. Tumors with a more advanced TNM stage (Stages III and IV) demonstrated a significantly higher lymphatic vascular area. Furthermore, lymphatic vessels were found to be significantly more numerous and larger in the peritumoral area compared to the intratumoral region. No significant correlation was found between the lymphangiogenesis parameters determined within and around the carcinomas with age, gender, tumor site and histologic differentiation.

Similar results have been reported by Filho et al.[20] In the current study, we observed significant association between PT-LVD with clinical stage and lymph node metastasis. No correlation was observed with age, gender, clinical $T$ stage, histological grade, and tumor site. Kreppel et al [30] reported a significantly lower 5 year overall survival in OSCC patients having high expression of podoplanin, as compared to those having a moderate or low expression $(p<0.001)$. Cervical lymph node metastasis was detected in $79 \%$ of the patients having high podoplanin expression, while only $22 \%$ of the patients with weak expression had metastasis $(p<0.001)$. In addition, podoplanin expression was significantly related to higher overall tumor stage. These results are similar to findings reported our study in terms of neck node metastasis and stage of tumor. We measured the podoplanin 
expression by D2-40 expression in peritumoral lymphatics, which probably is a more direct predictive evidence of lymphatic spread of cancer. Another difference in methodology is that Kreppel at al focused on the podoplanin expression on the primary tumor cells, which we believe is the indirect prognostic marker of lymph node metastasis.

Results of the current study coupled with review of the published literature suggest that there is a definite role of peritumoral lymphangiogenesis in the lymphatic spread of oral cancer. In the current study there was a significant association between PT-LVD and lymph node metastasis $(p=0.001)$. These cumulative data may be potentially implemented in clinical application, when deciding elective neck dissection in node negative T1/T2 patients undergoing a surgical tumor resection. Currently, a decision to perform elective neck dissection in NO patients is made on the primary tumor characteristics (site, size and depth of invasion) and neck imaging. High PT-LVD determined by D2-40 expression can assist in selecting patients with higher risk of occult metastasis.

PT-LVD assessed pre operatively along with primary tumor characteristics and imaging can be a more precise occult metastasis predictor. Several other markers that can predict lymphatic metastasis has been proposed. Epithelial mesenchymal transition (EMT), which is one of the common pathways for tumor invasion and metastasis, has been studied extensively. EMT markers SNAIL and SLUG have been shown to be associated with lymphatic metastasis. We believe that a combination of several predictor markers for neck metastasis applied simultaneously can have better predictive values for neck metastasis.

Conclusion: To conclude, Metastasis is a complex interplay of pathways which is yet incompletely understood. High PT-LVD is one such factor favoring metastasis. This study shows that high PT-LVD was significantly associated with lymph node metastasis and overall higher clinical stage. Addition of PT-LVD to currently used clinical tools and imaging may improve the prediction of lymph node metastasis in NO neck, and hence lead to better selection of patients for elective neck dissection in T1/T2 oral cancer. This therefore has the potential to modify neck treatment in oral cancer in future. Further prospective studies on larger sample size and correlation with overall survival would validate these findings further.

\section{References}

[1] Asthana S, Patil RS, Labani S. Tobacco-related cancers in India: A review of incidence reported from population-based cancer registries. Indian J Med Paediatr Oncol 2016. https://doi.org/10.4103/0971-5851.190357.

[2] Ferlay J, Soerjomataram I, Dikshit R, Eser S, Mathers $\mathrm{C}$, Rebelo $\mathrm{M}$, et al. Cancer incidence and mortality worldwide: Sources, methods and major patterns in GLOBOCAN 2012. Int J Cancer 2015;136:E359-86. https://doi.org/10.1002/ijc.29210.

[3] Balaram P, Sridhar H, Rajkumar T, Vaccarella S, Herrero R, Nandakumar A, et al. Oral cancer in Southern India: The influence of smoking, drinking, paan-chewing and oral hygiene. Int J Cancer 2002. https://doi.org/10.1002/ijc.10200.

[4] Cerezo L, Millan I, Torre A, Aragon G, Otero J. Prognostic factors for survival and tumor control in cervical lymph node metastases from head and neck cancer: A multivariate study of 492 cases. Cancer 2010;69:1224-34. https://doi.org/10.1002/cncr.2820690526.

[5] Pantvaidya G, Rao K, D'Cruz A. Management of the neck in oral cancers. Oral Oncol 2020;100:104476. https://doi.org/10.1016/j.oraloncology.2019.1044 76.

[6] Schöder $H$, Carlson DL, Kraus DH, Stambuk HE, Gönen $M$, Erdi $Y E$, et al. 18F-FDG PET/CT for detecting nodal metastases in patients with oral cancer staged NO by clinical examination and CT/MRI. J Nucl Med 2006;47:755-62.

[7] Pimenta Amaral TM, Da Silva Freire AR, Carvalho AL, Pinto CAL, Kowalski LP. Predictive factors of occult metastasis and prognosis of clinical stages I and II squamous cell carcinoma of the tongue and floor of the mouth. Oral Oncol 2004;40:780-6. https://doi.org/10.1016/j.oraloncology.2003.10.00 9.

[8] Köhler HF, Kowalski LP. A decision analysis model for elective neck dissection in patients with cT1-2 cNO oral squamous cell carcinoma. Acta Otorhinolaryngol Ital 2019;39:374-80. https://doi.org/10.14639/0392-100X-2101.

[9] Franchi A, Gallo O, Massi D, Baroni G, Santucci M. Tumor lymphangiogenesis in head and neck squamous cell carcinoma: A morphometric study with clinical correlations. Cancer 2004;101:973-8. https://doi.org/10.1002/cncr.20454.

[10] Neuchrist C, Erovic BM, Handisurya A, Fischer MB, Steiner GE, Hollemann D, et al. Vascular endothelial growth factor $C$ and vascular endothelial growth factor receptor 3 expression in squamous cell carcinomas of the head and neck. Head Neck 2003;25:464-74.

https://doi.org/10.1002/hed.10235.

[11] Mohammed RAA, Green A, El-Shikh S, Paish EC, Ellis 
IO, Martin SG. Prognostic significance of vascular endothelial cell growth factors $-A,-C$ and $-D$ in breast cancer and their relationship with angio- and lymphangiogenesis. Br J Cancer 2007;96:1092-100. https://doi.org/10.1038/sj.bjc.6603678.

[12] Schoppmann SF, Fenzl A, Nagy K, Unger S, Bayer G, Geleff S, et al. VEGF-C expressing tumor-associated macrophages in lymph node positive breast cancer: impact on lymphangiogenesis and survival. Surgery 2006;139:839-46.

https://doi.org/10.1016/j.surg.2005.12.008.

[13] Tobler NE, Detmar M. Tumor and lymph node lymphangiogenesis--impact on cancer metastasis. J Leukoc Biol 2006;80:691-6. https://doi.org/10.1189/jlb.1105653.

[14] He Y, Rajantie I, Pajusola K, Jeltsch M, Holopainen T, Yla-Herttuala $\mathrm{S}$, et al. Vascular endothelial cell growth factor receptor 3-mediated activation of lymphatic endothelium is crucial for tumor cell entry and spread via lymphatic vessels. Cancer Res 2005;65:4739-46. https://doi.org/10.1158/00085472.CAN-04-4576.

[15] Liang P, Hong JW, Ubukata H, Liu HR, Watanabe $Y$, Katano $\mathrm{M}$, et al. Increased density and diameter of lymphatic microvessels correlate with lymph node metastasis in early stage invasive colorectal carcinoma. Virchows Arch 2006;448:570-5. https://doi.org/10.1007/s00428-006-0166-9.

[16] Wirzenius $M$, Tammela T, Uutela $M$, He $Y$, Odorisio $\mathrm{T}$, Zambruno G, et al. Distinct vascular endothelial growth factor signals for lymphatic vessel enlargement and sprouting. J Exp Med 2007;204:1431-40.

https://doi.org/10.1084/jem.20062642.

[17] Hoshida T, Isaka N, Hagendoorn J, Di Tomaso E, Chen YL, Pytowski B, et al. Imaging steps of lymphatic metastasis reveals that vascular endothelial growth factor-C increases metastasis by increasing delivery of cancer cells to lymph nodes: Therapeutic implications. Cancer Res 2006;66:8065-75. https://doi.org/10.1158/00085472.CAN-06-1392.

[18] Van Der Auwera I, Van Den Eynden GG, Colpaert CG, Van Laere SJ, Van Dam P, Van Marck EA, et al. Tumor lymphangiogenesis in inflammatory breast carcinoma: A histomorphometric study. Clin Cancer Res 2005;11:7637-42. https://doi.org/10.1158/1078-0432.CCR-05-1142.

[19] Ohno F, Nakanishi H, Abe A, Seki Y, Kinoshita A, Hasegawa $Y$, et al. Regional difference in intratumoral lymphangiogenesis of oral squamous cell carcinomas evaluated by immunohistochemistry using D2-40 and podoplanin antibody: an analysis in comparison with angiogenesis. J Oral Pathol Med 2007;36:281-9. https://doi.org/10.1111/j.16000714.2007.00529.x.
[20] Longatto Filho A, Oliveira TG, Pinheiro C, de Carvalho MB, Curioni OA, da Cunha Mercante AM, et al. How useful is the assessment of lymphatic vascular density in oral carcinoma prognosis? World J Surg Oncol 2007;5. https://doi.org/10.1186/14777819-5-140.

[21] Parmar P, Marwah N, Parshad S, Yadav T, Batra A, Sen R. Clinicopathological Significance of Tumor Lymphatic Vessel Density in Head and Neck Squamous Cell Carcinoma. Indian J Otolaryngol Head Neck Surg 2018;70:102-10. https://doi.org/10.1007/s12070-017-1216-0.

[22] Miyahara M, Tanuma JI, Sugihara K, Semba I. Tumor lymphangiogenesis correlates with lymph node metastasis and clinicopathologic parameters in oral squamous cell carcinoma. Cancer 2007;110:128794. https://doi.org/10.1002/cncr.22900.

[23] Saharinen P, Tammela T, Karkkainen MJ, Alitalo K. Lymphatic vasculature: Development, molecular regulation and role in tumor metastasis and inflammation. Trends Immunol 2004;25:387-95. https://doi.org/10.1016/j.it.2004.05.003.

[24] Padera TP, Kadambi A, Di Tomaso E, Mouta Carreira C, Brown EB, Boucher Y, et al. Lymphatic metastasis in the absence of functional intratumor lymphatics.Science(80-)2002;296:1883-6. https://doi.org/10.1126/science.1071420.

[25] Reis-Filho JS, Schmitt FC. Lymphangiogenesis in tumors: What do we know? Microsc Res Tech 2003;60:171-80. https://doi.org/10.1002/jemt.10255.

[26] Kyzas PA, Geleff S, Batistatou A, Agnantis NJ, Stefanou D. Evidence for lymphangiogenesis and its prognostic implications in head and neck squamous cell carcinoma. J Pathol 2005;206:170-7. https://doi.org/10.1002/path.1776.

[27] Mehrotra R, Singh M, Kumar D, Pandey AN, Gupta RK, Sinha US. Age specific incidence rate and pathological spectrum of oral cancer in Allahabad. Indian J Med Sci 2003;57:400-4.

[28] Shenoi R, Devrukhkar V, Chaudhuri, Sharma BK, Sapre SB, Chikhale A. Demographic and clinical profile of oral squamous cell carcinoma patients: A retrospective study. Indian J. Cancer, vol. 49, Indian J Cancer; 2012, p. 21-6. https://doi.org/10.4103/0019-509X.98910.

[29] Yuan P, Temam S, El-Naggar A, Zhou X, Liu DD, Lee $\mathrm{JJ}$, et al. Overexpression of podoplanin in oral cancer and its association with poor clinical outcome. Cancer https://doi.org/10.1002/cncr.22061.

[30] Kreppel M, Scheer M, Drebber U, Ritter L, Zöller JE. Impact of podoplanin expression in oral squamous cell carcinoma: Clinical and histopathologic correlations. Virchows Arch 2010;456:473-82. https://doi.org/10.1007/s00428-010-0915-7. 
Tables

\begin{tabular}{|l|c|c|c|c|}
\hline \multicolumn{1}{|c|}{ Stage } & No.of patients & High PT-LVD & Low PT-LVD & \multirow{2}{*}{ P value } \\
\hline I & 10 & 8 & 2 & \multirow{2}{*}{0.033} \\
\hline II & 15 & 11 & 4 & \\
\hline III & 24 & 15 & 9 & \\
\hline
\end{tabular}

Table 1: Site distribution of Oral SCC at presentation $(n=72)$

\begin{tabular}{|l|c|}
\hline \multicolumn{1}{|c|}{ Site of oral cancer } & Percentage of patients \\
\hline Tongue & 33.3 \\
\hline Buccal mucosa & 58.3 \\
\hline Lip & 4.1 \\
\hline Maxillary alveolus and hard palate & 4.1 \\
\hline
\end{tabular}

Table 2: Association between degree of tumor differentiation and peritumoral lymphatic density

\begin{tabular}{|l|c|c|c|c|}
\hline Degree of differentiation & No. of patients & High PT-LVD & Low PT-LVD & P value \\
\hline Well diff. & 23 & 13 & 10 & \multirow{2}{*}{0.177} \\
\hline Mod. Diff. & 48 & 28 & 20 & \\
\hline Poorly diff. & 1 & 1 & 0 & \\
\hline
\end{tabular}

Table 3: Association of clinical neck node status and PT-LVD

\begin{tabular}{|l|c|c|c|c|}
\hline $\begin{array}{c}\text { Clinical lymph node } \\
\text { status }\end{array}$ & No. of patients & High PT-LVD & Low PT-LVD & P value \\
\hline $\mathrm{N}+$ & 33 & 28 & 05 & \multirow{2}{*}{0.001} \\
\hline $\mathrm{N}-$ & 39 & 14 & 25 & 0.001 \\
\hline
\end{tabular}

Table 4: Association of clinical stage and PT-LVD

Table 5: Association of primary tumor and PT-LVD

\begin{tabular}{|l|c|c|c|c|}
\hline \multicolumn{1}{|c|}{ Group } & No.of patients & High PT-LVD & Low PT-LVD & $\begin{array}{c}\text { P value (chi- } \\
\text { square test) }\end{array}$ \\
\hline I (T1\&T2) & 36 & 25 & 11 & \multirow{2}{*}{0.538} \\
\hline II (T3\&T4) & 36 & 17 & 19 & \\
\hline
\end{tabular}


Table 6: Association of primary tumor site and PT-LVD

\begin{tabular}{|l|l|l|l|l|}
\hline Site & No. of patients & High PT-LVD & Low PT-LVD & P value \\
\hline Tongue & 24 & 17 & 7 & \multirow{2}{*}{0.373} \\
\cline { 1 - 4 } Buccal mucosa & 42 & 21 & 21 & \\
\hline Lip & 3 & 2 & 1 & \\
\cline { 1 - 4 } Palate & 3 & 2 & 1 & \\
\hline
\end{tabular}


Figure legends:

Fig. 1 IHC, D2-40 (x200) shows peritumoral lymphatic vessels (thin arrow) stained specifically with D2-40 and negatively stained blood vessels (thick arrow)

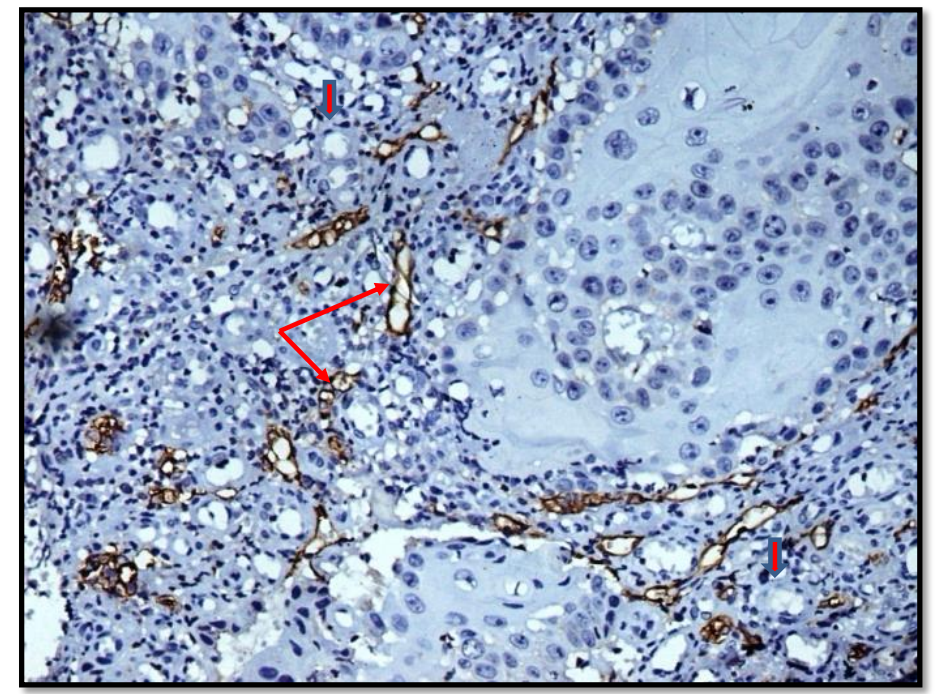

IHC, D2-40 (x200) shows peritumoral lymphatic vessels (thin arrow) stained specifically with D2-40 and negatively stained blood vessels (thick arrow) 
Fig. 2 IHC, D2-40 (x100) high peritumoral lymphovascular density shows staining positively for D2-40 in squamous cell carcinoma buccal mucosa. High power view of the same shown in the inset (x200)

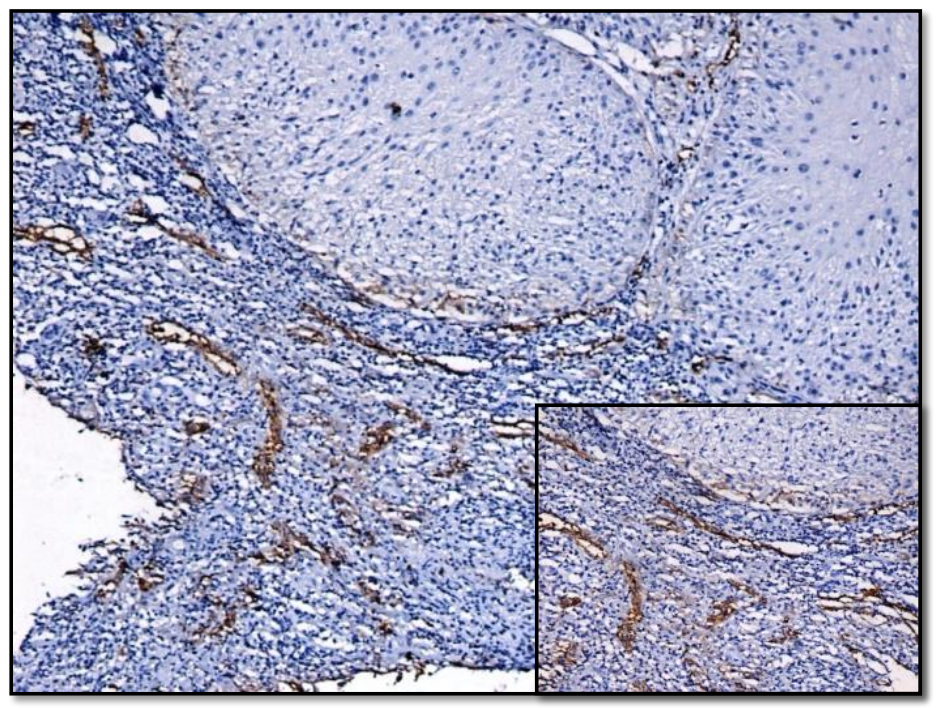

IHC, D2-40 (x100) high peritumoral lymphovascular density shows staining positively for D2-40 in squamous cell carcinoma buccal mucosa. High power view of the same shown in the inset (x200) 
Fig. 3 IHC, D2-40 (x100) shows high peritumoral lymphatic density in squamous cell carcinoma buccal mucosa. Inset high power view of same (x200) show lymphatic vessels stained positivity for D2-40

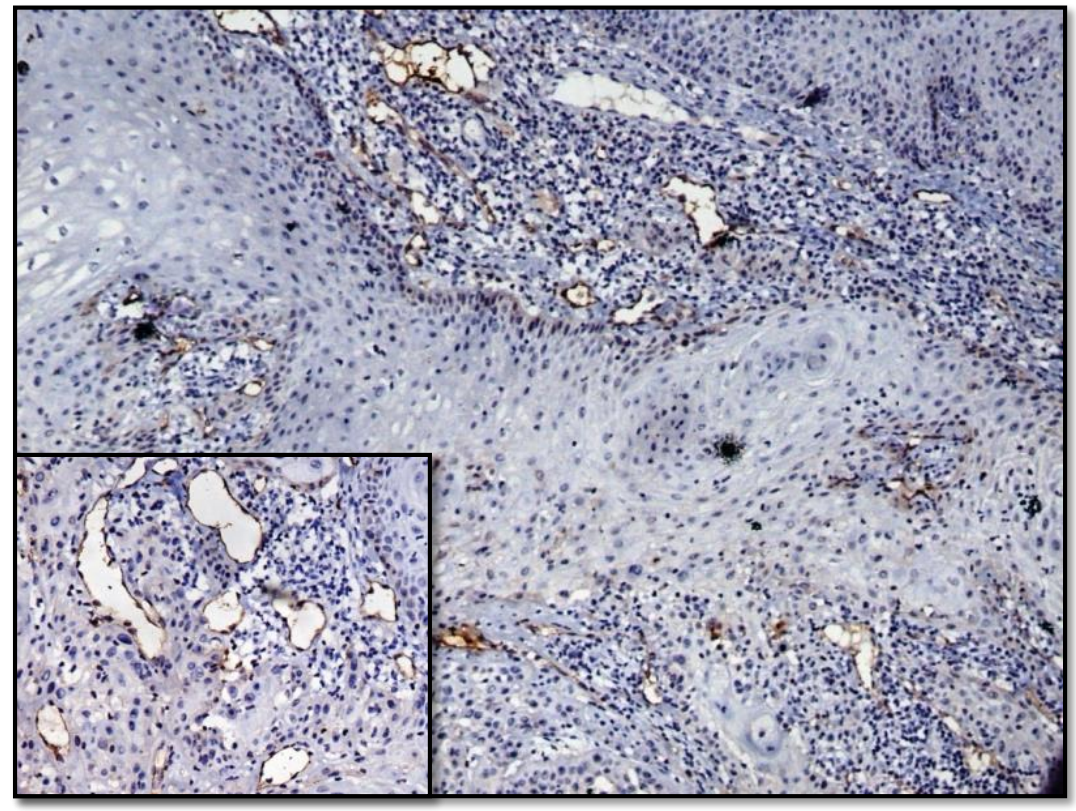

IHC, D2-40 (x100) shows high peritumoral lymphatic density in squamous cell carcinoma buccal mucosa. Inset high power view of same $(x 200)$ show lymphatic vessels stained positivity for D2-40 